\title{
Sobre el Calentamiento Global en la isla Rey Jorge: procesos y evidencias en el Glaciar Wanda y su entorno
}

\author{
Francisco José Ferrando Acuña ${ }^{1}$ \\ Rosemary Vieira ${ }^{2}$ \\ Kátia Kellem da Rosa ${ }^{2}$ \\ ${ }^{1}$ Departamento de Geografía - Universidad de Chile. \\ ${ }^{2}$ NUPAC - Universidade Federal do Rio Grande do Sul, Br. \\ E-mails: \\ fferrand@uchile.cl \\ rosenupac@yahoo.com.br \\ katiakellem@yahoo.com.br
}

\section{RESUMEN}

Se analizan antecedentes geológicos, volcánicos, meteorológicos y vegetacionales, tanto fósiles como actuales, con el objeto de encontrar respuestas a la dinámica de los glaciares de la isla Rey Jorge, Archipiélago de las Shetlands del Sur, con especial énfasis en los aspectos de la dinámica glaciológica presentados por el Glaciar Wanda, ubicado en la Ensenada Martel, Seno Almirantazgo, así como por aspectos relevantes evidenciados en el Glaciar Ecology, localizado dentro del mismo Seno. Los análisis geomorfológicos y glaciológicos realizados en terreno, los resultados morfológicos de los sedimentos glaciales, los hallazgos de vegetales fósiles, así como la interpretación de los antecedentes volcánicos de la península Antártica, de las formas residuales y piroclastos de este origen, junto a la experimentación directa de condiciones de tiempo con precipitaciones líquidas sobre los glaciares, las respuestas hidrológicas generadas y el desarrollo de cubiertas vegetales más que pioneras, entre otros elementos de juicio, revelan un complejo panorama evolutivo de las condiciones glaciológicas en la isla, en las que se aparecen como fundamentales el alzamiento de las temperaturas y la irrupción de precipitaciones líquidas en un significativo número de días por año, hecho que tiene precedentes, a lo que en el plano de lo teórico por ahora, se suma la posible influencia de calor latente derivado del contexto volcánico. Hoy en día, en el caso del Glaciar Wanda se evidencian claros efectos de este escenario en su balance de masa, registrándose desde claras evidencias de retroceso de su frente hasta momentos de fusión acelerada y generación de pequeños torrentes y lagunas, algunas represadas discontinuamente por morrenas frontales dentro del ámbito litoral y de carácter persistente, así como otras de carácter estacional dentro del ambiente glacial.

Palabras clave: Glaciodinámica, calentamiento, lluvias, fósiles vegetales, vegetación actual, contexto volcánico, repuesta hidrodinámica, consunción, lagunas.

\begin{abstract}
Geologic, geomorphologic and volcanic aspects, meteorological data and vegetation, both fossil and current, are considered in order to find answers to the dynamics of King George Island glaciers, into the South Shetlands Islands, with special emphasis on aspects of the glaciological dynamics submitted by Wanda glacier located in Martel inlet, Almirantazgo Sound, as well as by relevant aspects shown by the Ecology glacier, located at the neighborhood. Glaciological and geomorphologic field analysis carried out, the morphological results of glacial sediments samples at laboratory, plant fossils finds, as well as the interpretation of volcanic history of the Península Antártica, joint with volcanic residual forms and piroclastic materials, also the direct experience of rain over ice, the hydrological responses generated, and the development of not pioneer plants, among other elements, reveal a complex island glaciological
\end{abstract}


evolution. So, temperature rate increasing and the occurrence of rain in a significant number of days per year, scenery that seems not new in the past of the island, and the possible influence of latent heat derived from the volcanic context means in the case of Wanda glacier clear negative effects on his mass balance, registering from clear evidence of front retreat to moments of accelerated melting and generation of small torrents and lagoons, some of them with persistent character discontinuously dammed by front moraines at the coast, as well as other of seasonally formed into the glacial environment.

Key words: Glaciological dynamic, warming, rain, plant fossils, actual vegetation, volcanic context, hydrodynamic response, melting, lagoons.

\section{INTRODUCCIÓN}

En relación con la discusión global sobre el calentamiento climático, la realización de una de las actividades de campo al Glaciar Wanda, ubicado en la Ensenada Martel del Seno Almirantazgo, Isla Rey Jorge (Shetland del Sur), a una latitud de $62^{\circ} 07^{\prime}$ Sur y $58^{\circ} 21^{\prime}$ Oeste (Figura $N^{o} 1$ ), con motivo del proyecto "Respuesta de la Criosfera al
Cambio Climático", apoyado por el NUPAC (Núcleo de Pesquisas Antárticas e Climáticas-UFRGS) y el PROANTAR (Programa Antártico Brasilero), ha permitido a un equipo de investigadores brasileño-chileno observar directamente en terreno diversas características de la dinámica de este cuerpo de hielo, así como de algunos cercanos, las cuales redundan en un balance de masa visiblemente negativo.

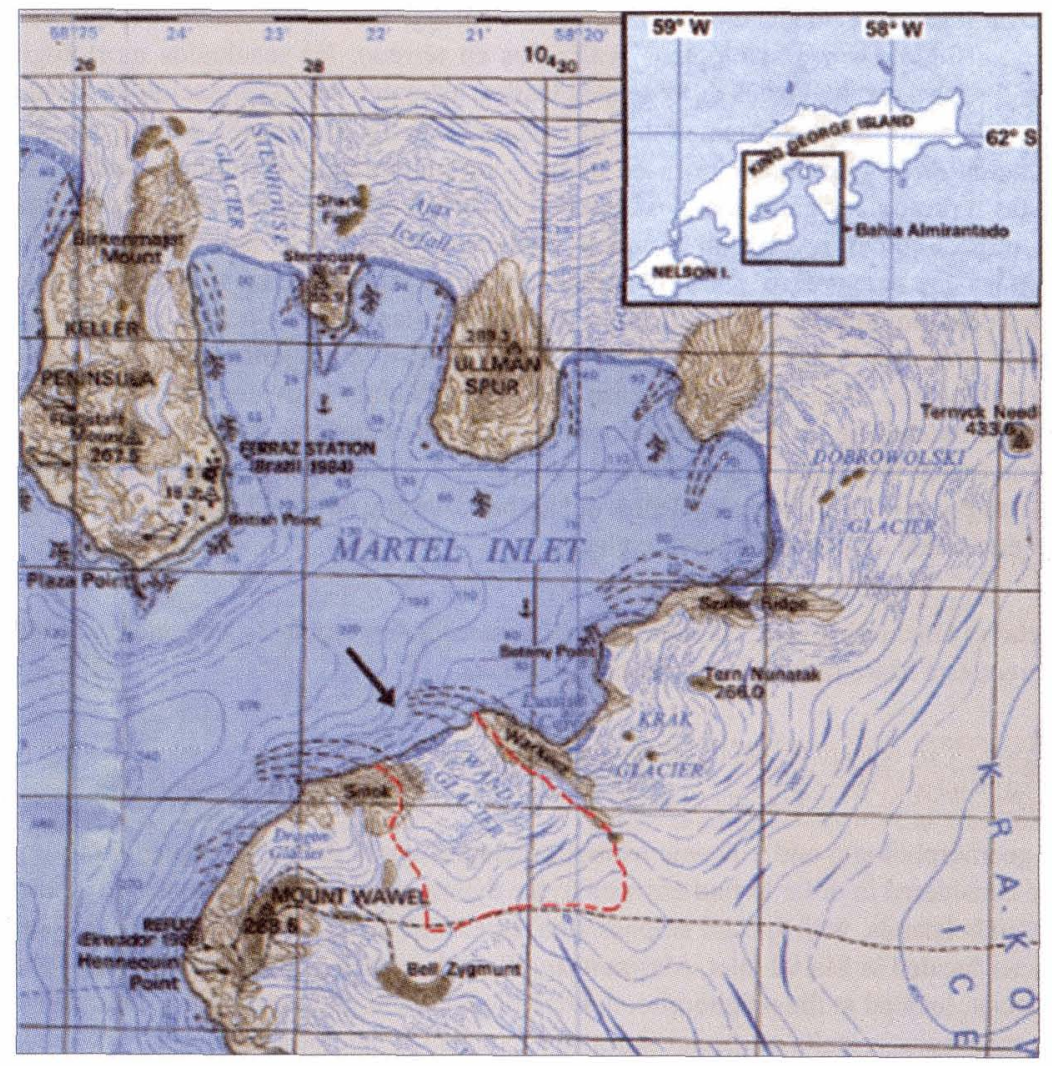

Figura $\mathbf{N}^{\circ}$ 1. Mapa de Ubicación del Glaciar Wanda. 
Paralelamente, se analizan series de datos pluviométricos, información de variaciones de temperatura, y se consideran los resultados de estudios geomorfológico-sedimentológicos que vienen a avalar la relación entre la dinámica del glaciar y lo que está aconteciendo en términos del actual proceso de calentamiento climático, el cual tiene precedentes en la historia geológica de la península Antártica.

\section{ANTECEDENTES SOBRE LA ISLA REY JORGE}

\section{Naturaleza geológico-volcánica de la isla}

Aunque en la isla Rey Jorge no se han descrito volcanes, su naturaleza litológica, la existencia de capas de lava, brechas volcánicas y materiales piroclásticos de naturaleza explosiva, por una parte, y la cercanía a centros volcánicos como Isla Penguin e Isla Decepción por otra, dentro de los al menos once centros volcánicos con actividad Pleistoceno tardío a Holocénica conocida o sospechada (Kraus, S., 2008), hacen evidente el pasado volcánico de la isla tanto en su génesis como en su evolución.

Esto viene a ser corroborado por la presencia de fragmentos piroclásticos altamente vesiculares $y$, también, por la existencia de formaciones rocosas que constituyen posibles chimeneas volcánicas denudadas (Ternick Needle; Czajkovsky Needle; Arctowsky Needle). (Fotos 1 y 2 ).

Estos posibles neck's cuentan con otros exponentes en el área, tal como la Sail Rock ubicada al SW de la Isla Decepción, la que es descrita como la parte superior remanente de un volcán sumergido (delgada chimenea de cerca de $30 \mathrm{~m}$ de altura- Kraus, 2008).
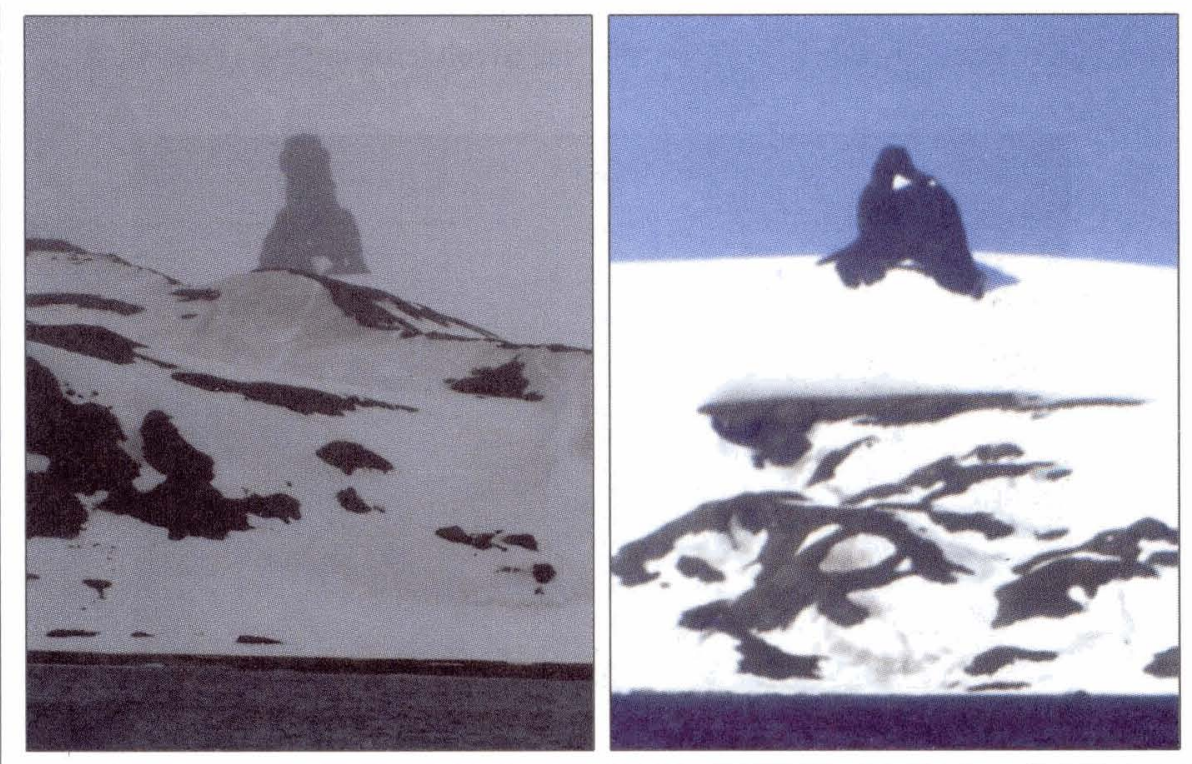

Fotos 1 y 2. Czajkovsky Needle y Ternyck Needle. Posibles chimeneas volcánicas.

\section{Evolución glacial pasada}

La historia glacial de la Isla Rey Jorge revela varios períodos de glaciación y deglaciación a partir de la máxima extensión registrada entre 20.000 y 18.000 años BP. Más recientemente, los estudios paleoclimáticos realizados por Yoon et al. (2000) sugieren un clima más temperado y húmedo entre 4.000 y 2.700 años B.P., período en el 
que podría haberse desarrollado vegetación leñosa. Restos de troncos y hojas fósiles encontrados durante esta investigación en rocas de diferente origen y edad del lecho rocoso del Glaciar Wanda, viene a agregar una prueba más a la existencia de estadios climáticos menos fríos.

\section{¿Historia nueva?}

Esta situación no es algo nuevo en la historia paleo-climática de la isla Rey Jorge. En el caso del Glaciar Wanda, las investigaciones de campo realizadas permitieron reconocer las huellas geomorfológicas de la existencia de, al menos, tres estadios de avance dentro de un proceso holocénico de evolución regresiva general, a saber: El glaciar principal con una difluencia mayor; El glaciar principal con dos difluencias menores; Solo el glaciar principal (ver Rosa et al, 2008b), lo cual plantea claramente la ocurrencia de oscilaciones climáticas más o menos prolongadas.
Por otra parte, Torres et al. (2000) señala que el hallazgo de maderas petrificadas (Nothofagus), impresiones de hojas, polen y esporas encontrados en la Península Antártica permite deducir el paso de ambientes cálidos y húmedos del Cretácico a temperado-fríos hacia fines del Terciario, lo que habría iniciado las glaciaciones que terminaron en el Cuaternario.

Corroborando lo anterior, en el lecho del Glaciar Wanda, el aparecimiento en superficie de sedimentos subglaciales y de capas de rocas sedimentario-volcánicas del sustrato geológico producto del retroceso de los hielos ha dejado al descubierto la existencia de flora fosilizada de edades muy distintas.

Durante la campaña de terreno de diciembre 2007, en este sector se encontraron tanto troncos y hojas fosilizadas, completamente mineralizadas (Foto 3), como otros fragmentos que aún conservan su naturaleza orgánica, aunque aparentemente carbonizada por acción de los procesos volcánicos que las envolvieron en lava (Foto 4).

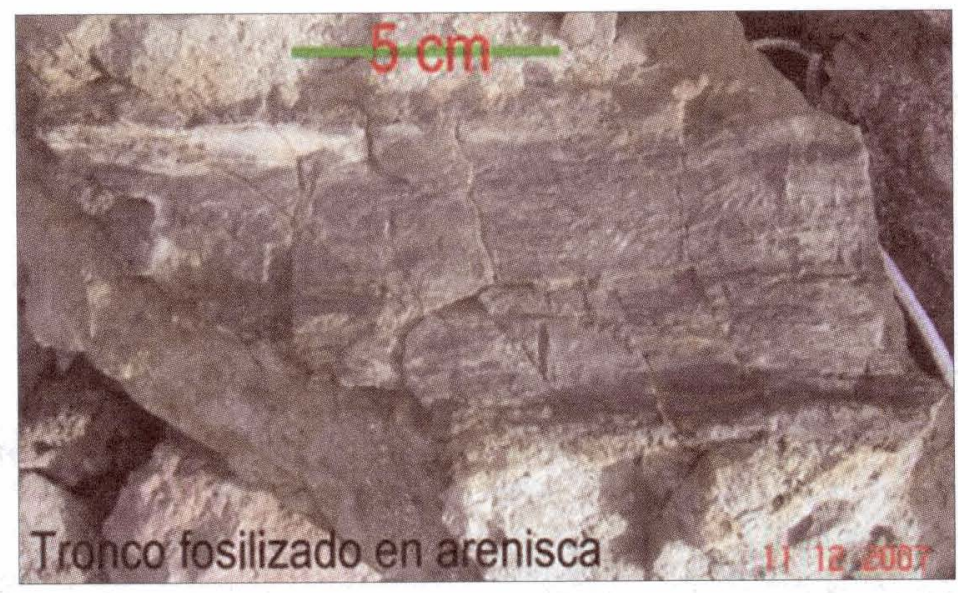

Foto 3. Fósil de tronco completamente mineralizado en un fragmento de arenisca.

\section{Otros indicadores}

La existencia de cordones litorales de cantos rodados (Pallas et al., 1995 y 1997) como los encontrados durante esta investigación en las inmediaciones del Glaciar Ecology a algunos metros por sobre los alcances de los efectos de la acción marina actual (Foto 5), así como el que este tipo de cordones se forme durante épocas en que el mar no se congela en invierno (Santana et al., 2008), constituyen otro elemento de juicio que 
permite sostener la ocurrencia de períodos templados con mayor frecuencia de preci- pitaciones líquidas, posiblemente desde el pleistoceno.

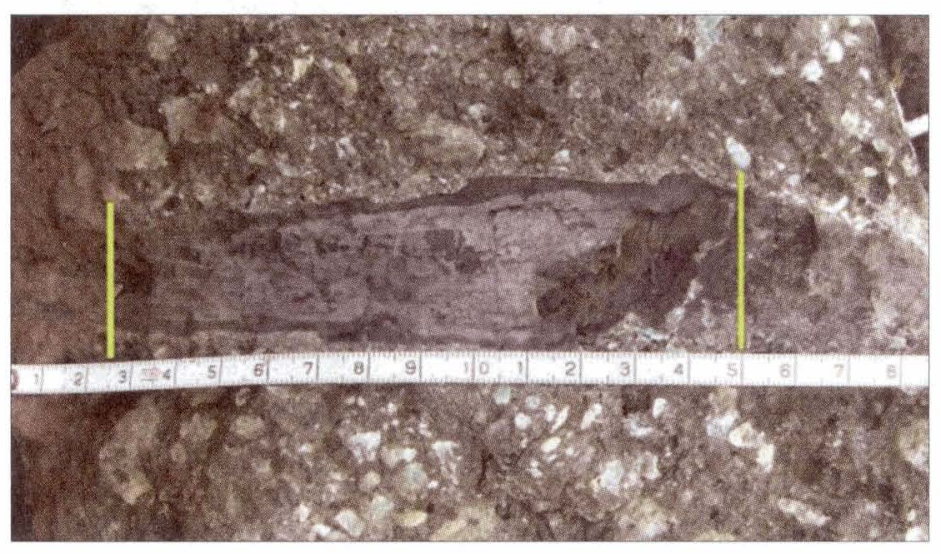

Foto 4. Restos de tronco carbonizado de $12,5 \times 3,0 \mathrm{~cm}$. en bloque de brecha volcánica.

La edad pleistocénica supuesta para estos cordones, otrora litorales, tiene relación con el origen del desnivel respecto de la costa actual. En este sentido, se pueden invocar posibles solevantamientos glacio-isostáticos de la isla Rey Jorge, dada la reducción gradual de la calota de hielo que la cubría durante las glaciaciones por el aumento paulatino de las temperaturas, tal como se ha establecido por ejemplo para Italia (Antonioli, 2007) y para la Península Escandinava (Morner, 1980), entre otros. (Lambeck et al., 2004). También, según Berrocoso et al., (2008) podrían estar involucrados mecanismos tectónicos.

\section{Los registros meteorológicos actuales}

Respecto de hechos que revelan calentamiento reciente, la base de datos meteorológicos de la Estación Brasileña Comandante Ferraz deja en evidencia que desde 1986 y, a decir de los antecedentes entregados por antiguos investigadores, desde hace unos 30 años que en el sector se está registrando un número considerable de días al año en que se producen precipitaciones líquidas sobre las superficies de nieve y hielo. (Ver Tabla $\mathrm{N}^{\circ} 1$ y Gráfico $\mathrm{N}^{\circ} 1$ ).

Conforme a estos datos, se verifica que, en el período de la serie de datos, los días con precipitaciones líquidas han representado porcentajes entre 22,7 y 59,2 respecto del total de días con precipitaciones, lo cual es altamente significativo en términos de los efectos sobre el balance de masa de los glaciares de la Isla Rey George.

Evolutivamente, más allá del hecho que sólo en los años 1987, 1992 y 1999, la precipitación líquida superó el 50\% del total, es claro que en la primera mitad del período registrado (1986-1996) el porcentaje de días con lluvia es superior en promedio al de la segunda mitad (1997-2007), variando de 44,71 a $36,64 \%$. Esta variación, dado lo corto del registro, sólo se puede considerar como una oscilación menor que en modo alguno resta trascendencia a la ocurrencia significativa de precipitaciones líquidas sobre hielo a $62^{\circ}$ de latitud sur. 


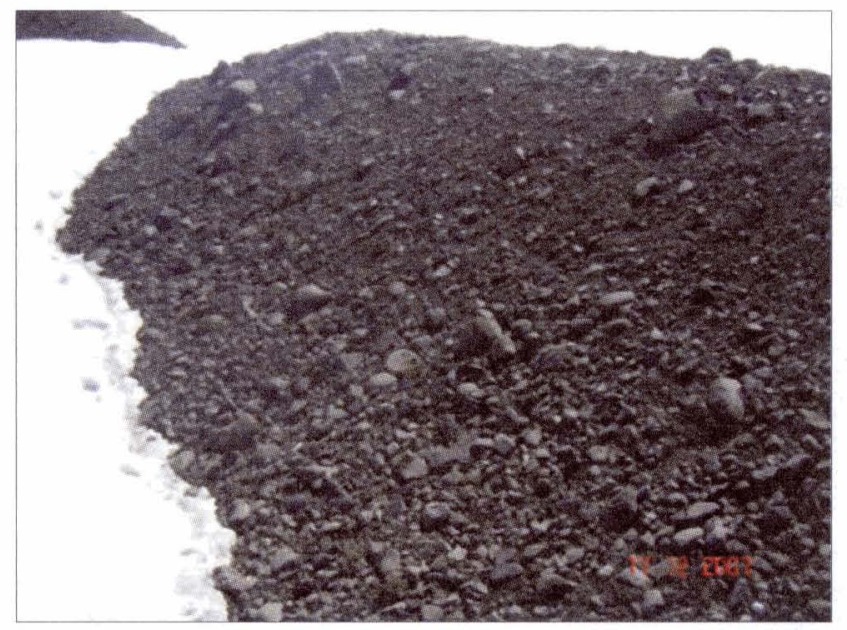

Foto 5. Cordón de rodados en el costado NE del Glaciar Ecology.

\section{Tendencias térmicas}

Se sostiene que la Península Antártica es una de las regiones del mundo más afectadas por el calentamiento global, dado que el aumento de las temperaturas atmosféricas supera hasta en seis veces el promedio mundial (Vaughan et al., 2001; Vaughan et al.,2003).

Múltiples estudios -King (1994); King y Harangozo (1998); Marshal y otros (2002), King y Comiso (2003) - han mostrado el fuerte calentamiento que en la Península Antártica ha estado ocurriendo desde mediado del siglo XX, hecho que no tiene paralelo con lo que ocurre en el resto del planeta.

Paralelamente, de acuerdo a Ferron et al. (2001), la temperatura media del aire en la isla Rey Jorge habría subido en $1,08^{\circ} \mathrm{C}$ en el período 1947-1995. Los estudios de es- pecialistas norteamericanos han concluido que en la región Antártica las temperaturas han aumentado cerca de $2,5^{\circ}$ en los últimos 50 años. Ya en 1978, J. Mercer anticipaba lo que comenzaría a ocurrir sólo 10 años después en las plataformas de hielo de la Antártica producto del calentamiento global. Tal es el caso de la desintegración de las plataformas flotantes de hielo Wordie en 1989 (Vaughan, 1993), Larsen A en 1995 y Larsen B en el año 2002, fenómenos que arrojaron millones de kilómetros cúbicos de agua dulce al mar.

Lo señalado encuentra su correspondencia en los datos de la Estación Cdte. Ferraz (Br) en cuanto a que en el período 1986-2006 tanto las temperaturas medias anuales como las medias mínimas y las medias máximas han experimentado una tendencia ascendente de $1,2^{\circ} \mathrm{C}$ en sólo 20 años (Gráfico 2). 
Tabla $\mathbf{N}^{\circ}$ I. Precipitaciones registradas en la estación meteorológica de la Base Comandante Ferraz (Br). Período 1986 - 2007.

\begin{tabular}{|c|c|c|c|c|}
\hline Años & Dias nieve & Dias Iluvia & $\begin{array}{l}\text { Total dias } \\
\text { con Pp }\end{array}$ & $\begin{array}{l}\text { \% Dias lluvia sobre } \\
\text { total dias con Pp }\end{array}$ \\
\hline 1986 & 77 & 48 & 125 & 38,4 \\
\hline 1987 & 86 & 108 & 194 & 55,7 \\
\hline 1988 & 156 & 106 & 262 & 40,5 \\
\hline 1989 & 156 & 131 & 287 & 45,6 \\
\hline 1990 & 133 & 102 & 235 & 43,4 \\
\hline 1991 & 88 & 85 & 173 & 49,1 \\
\hline 1992 & 64 & 93 & 157 & 59,2 \\
\hline 1993 & 169 & 102 & 271 & 37,6 \\
\hline 1994 & 197 & 144 & 341 & 42,2 \\
\hline 1995 & 142 & 102 & 244 & 41,8 \\
\hline 1996 & 167 & 104 & 271 & 38,4 \\
\hline 1997 & 160 & 82 & 242 & 33,9 \\
\hline 1998 & 151 & 90 & 241 & 37,3 \\
\hline 1999 & 79 & 99 & 178 & 55,6 \\
\hline 2000 & 127 & 55 & 182 & 30,2 \\
\hline 2001 & 235 & 89 & 324 & 27,5 \\
\hline 2002 & 123 & 67 & 190 & 35,3 \\
\hline 2003 & 107 & 70 & 177 & 39,5 \\
\hline 2004 & 106 & 83 & 189 & 43,9 \\
\hline 2005 & 116 & 86 & 202 & 40,6 \\
\hline 2006 & 162 & 93 & 255 & 36,5 \\
\hline 2007 & 177 & 52 & 229 & 22,7 \\
\hline
\end{tabular}

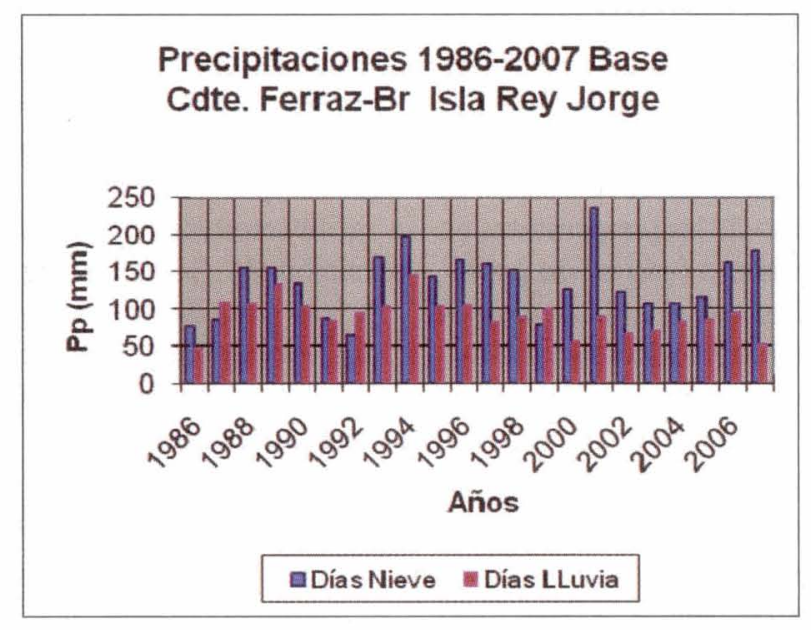

Gráfico $N^{0} 1$. Precipitaciones sólidas y líquidas. Datos de la Of. Meteorológica de la Base Comandante Ferraz, Brasil, Antártica. 


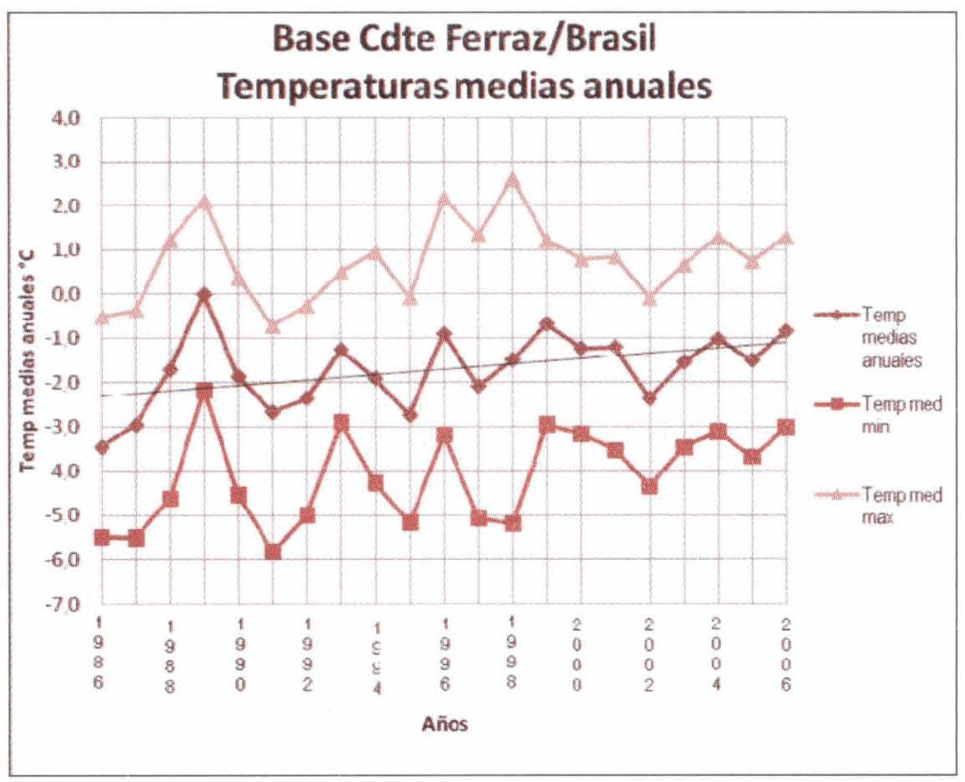

Gráfico 2. Evolución de las temperaturas medias anuales, mínimas medias y máximas medias en el período 1986-2006. Datos de la Of. Meteorológica de la Base Comandante Ferraz, Brasil, Antártica.

A lo anterior se agrega el hecho que las temperaturas máximas absolutas registradas en la oficina meteorológica de la base brasileña variaron de $9,4^{\circ} \mathrm{C}$ a $14,9^{\circ} \mathrm{C}$ para el mismo período, siendo el promedio de $10,5^{\circ} \mathrm{C}$. Por su parte, el promedio de las temperaturas máximas medias alcanzó los $0,7^{\circ} \mathrm{C}$ para el período registrado.

Respecto de otros registros térmicos en la Isla Rey Jorge, los análisis del comportamiento de las temperaturas en la Estación meteorológica de la Base Pdte. Eduardo Frei (Ch) indican que, entre 1970 y 2004, la tendencia al aumento de las temperaturas es muy clara en las mínimas (oscilación positiva de más de $3^{\circ} \mathrm{C}$ ) y no así en las temperaturas máximas, las cuales muestran incluso una leve disminución a partir de la década de los ' 80 .

El comportamiento de la temperatura media anual a través de los años de registro de otras estaciones cercanas a la Base Frei, como Bellingshuasen (1951-2004) y Faraday (1970-2002), también revelan un considerable calentamiento del aire superficial
( $\approx 0.5$ y $2.5^{\circ} \mathrm{C}$, respectivamente). Sin embargo, debe considerarse lo aseverado por King y Comiso (2003) en términos de que esta tendencia de las temperaturas es más evidente en el sector noroeste de la península, lo cual es corroborado por Turner et al. (2005), quien afirma que ello no está ocurriendo en el resto de la Antártica.

En todo caso, lo señalado conduce a la existencia de períodos intra-anuales de temperaturas por sobre $0^{\circ} \mathrm{C}$, lo que sumado a la ocurrencia de precipitaciones líquidas sobre los cuerpos de hielo lleva, como se observa en diversos registros fotográficos, a la formación de múltiples lagunas sobre ellos. A esta pérdida de masa por reducción del espesor derivado tanto de las lluvias como de las temperaturas, se suma el evidente aumento de la evaporación y la sublimación, así como las presiones y efectos del oleaje marino y las mareas en el caso de los tidal glaciers y las plataformas.

Pero este fenómeno no está afectando sólo a este tipo de glaciares, ya que en el caso de las lenguas de los glaciares litorales no 
conectados en su frente con plataformas de hielo (Ej.: Glaciar Ecology, Glaciar Wanda) está ocurriendo algo similar en términos de adelgazamiento y pérdida de masa, es decir, se trataría de una situación generalizada a todos los cuerpos glaciarios litorales, los que tienen en común su baja altura y su exposición a los efectos oceánicos.

En el escenario térmico señalado precedentemente, y corroborando lo anterior, en el Glaciar Wanda se observa una rápida res- puesta de los procesos de consunción ante la ocurrencia de días con temperaturas ligeramente por sobre $0^{\circ} \mathrm{C}$, los que pueden presentarse con o sin ocurrencia de precipitaciones. Ello se traduce, como pudo observarse en terreno, en activos procesos de fusión, despeje local del lecho rocoso, formación de cursos de agua y aparecimiento de lagunas, las que se mantienen o crecen gradualmente una vez formadas durante estos episodios del verano polar (Foto 6).

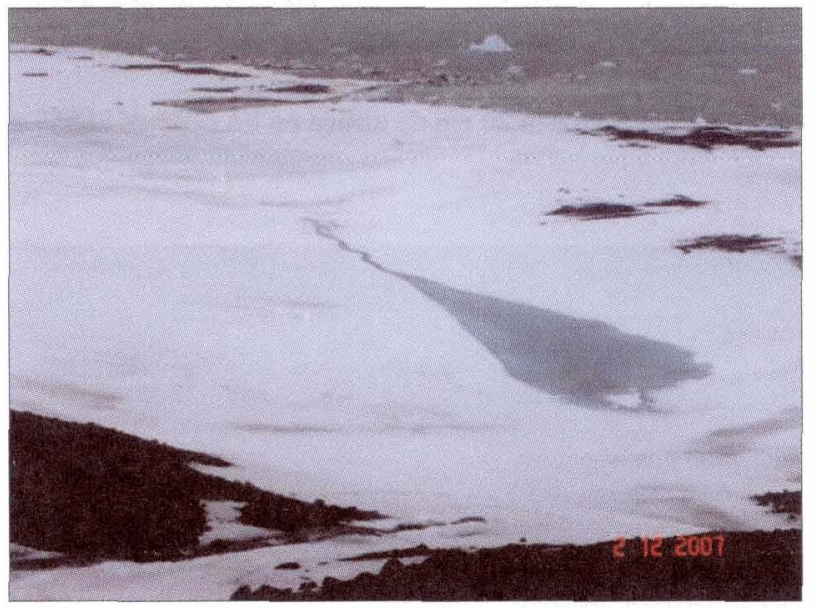

Foto 6. Laguna de fusión en el costado NE del Glaciar Wanda después de un día de lluvia. Se aprecia una laguna cubierta en la parte baja, cerca del mar.

Es claro que la ocurrencia conjunta de precipitaciones líquidas acompañadas de temperaturas algunos grados por sobre $0^{\circ} \mathrm{C}$ constituye un escenario que desencadena violentos procesos de fusión de hielo y nieve, como se pudo constatar durante la campaña antártica 2007.

Con el objeto de cuantificar y realizar un seguimiento de estos procesos, principalmente el adelgazamiento del Glaciar Wanda, se inició como parte de la investigación la instalación de balizas que permitirán seguir y registrar la magnitud del proceso durante las campañas siguientes.

\section{Evidencias vegetacionales}

Otro hecho que viene a indicar este temperamiento del clima en el sector es la presencia no sólo de importantes manchones de musgos y líquenes de diversas especies (Foto 7), sino que también de algunas especies herbáceas resistentes al frío que vienen a formar pequeños sectores de praderas (Foto 8). A este respecto, y como ejemplo, en el sector entre el Glaciar Ecology y la Base Polaca Arktowsky, existe una pradera de varias hectáreas, lo que viene a testimoniar el hecho de que no se trata de una situación puntual dentro de la isla. 
(6 070

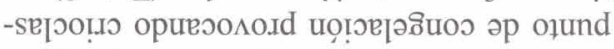

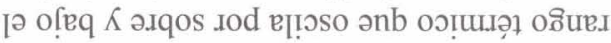

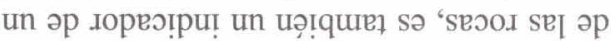

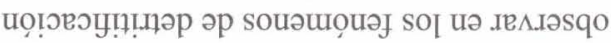

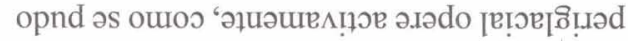

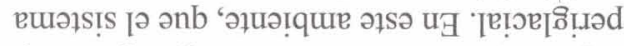

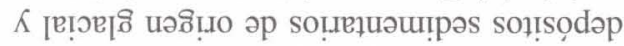

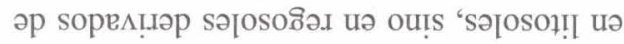

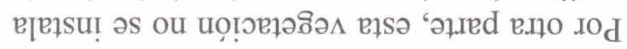

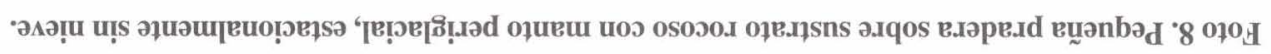

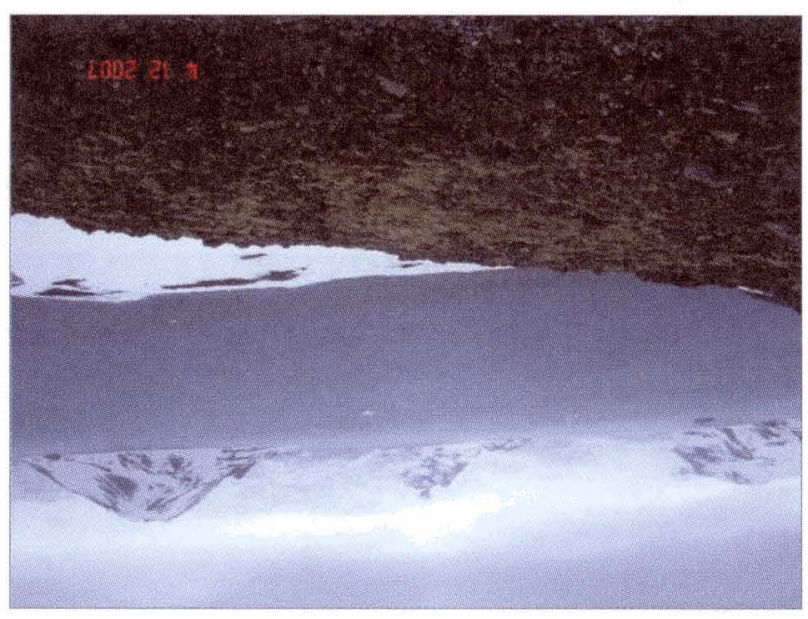

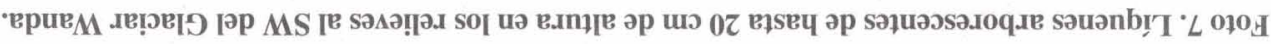

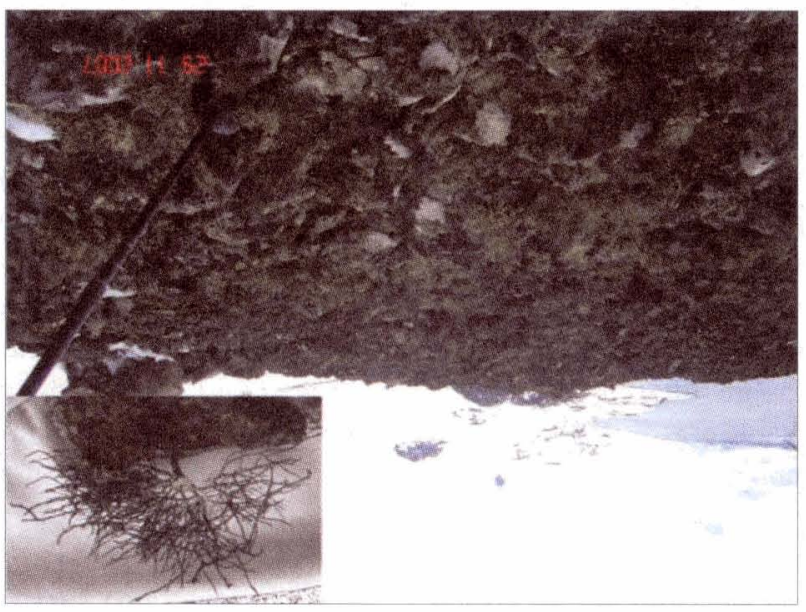




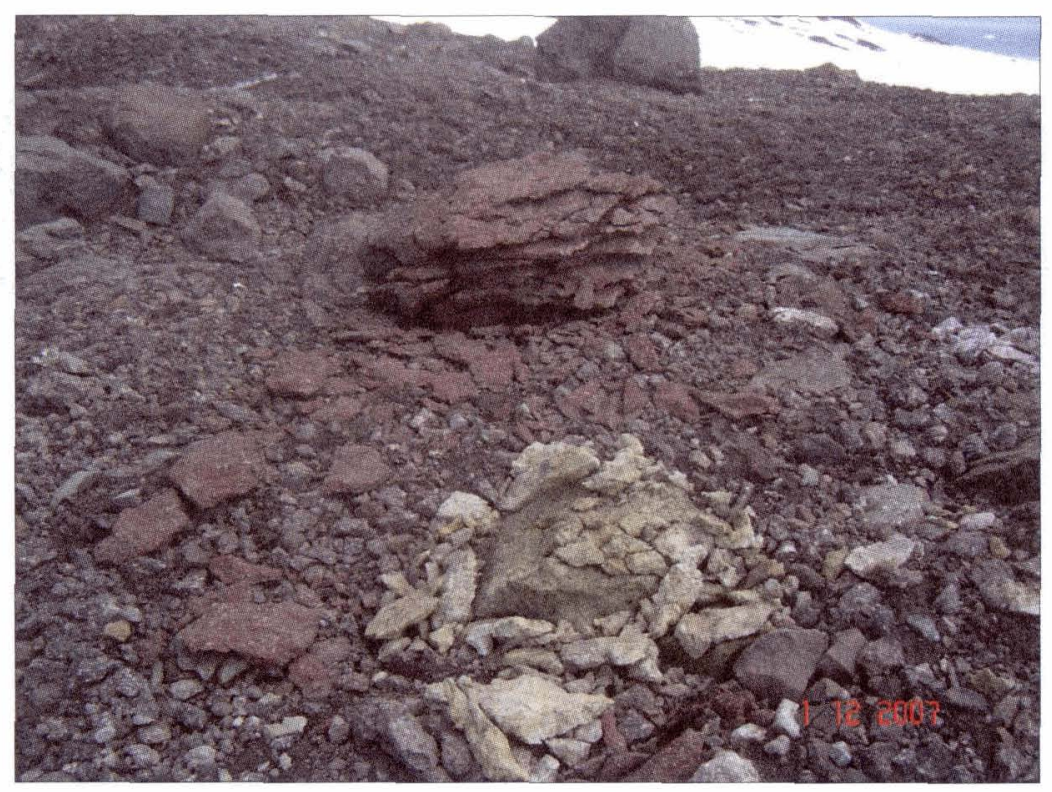

Foto 9. Ejemplos del accionar del sistema periglacial en materiales morrénicos laterales del Glaciar Wanda.

\section{Los efectos de las precipitaciones líquidas}

La ocurrencia de precipitaciones líquidas sobre el hielo y la nieve, en condiciones ambientales sobre $0^{\circ} \mathrm{C}$, hace que estos fundan rápidamente, apareciendo en forma progresiva sectores de roca o de sedimentos subglaciales expuestos, con abundante formación de barro. Dichos eventos pluviométricos vienen a saturar los depósitos morrénicos de tal forma que con frecuencia se aprecia la formación de flujos detríticos de algunos metros de alcance a partir de sus caras más abruptas. (Fotos 10 y 11)

Pero además, este fenómeno está afectando directamente los cuerpos de hielo generando un considerable adelgazamiento o pérdida de espesor de ellos en las últimas décadas. Este hecho que se observa claramente en el Glaciar Wanda, ya ha sido documentado en el caso del Glaciar Ecology (Vieira et al., 2005; Rosa et al., 2006). En este sentido,
Simoes et al., (1999) señala que las masas de hielo de las islas del Archipiélago de las Shetland del Sur, por su ubicación geográfica y poco espesor relativo, presentan condiciones que favorecen las respuestas rápidas frente a cambios climáticos.

El agua de la lluvia, y la de fusión producida por el efecto de la primera y de las temperaturas sobre $0^{\circ} \mathrm{C}$ durante estos eventos, no sólo provoca derretimiento de la superficie de nieve y neviza sobre el glaciar, sino que, como pudo observarse, penetra a través de las grietas, provoca ensanchamiento de las mismas, y genera flujos de circulación interna y subglacial, con lo que la presencia de agua va minando estos glaciares periantárticos y derritiéndolos externa e internamente. Se trata de un mecanismo que es comparable con los procesos de consunción propios de glaciares temperados ubicados mucho más al norte, en la Cordillera de los Andes de Chile centro-sur. 


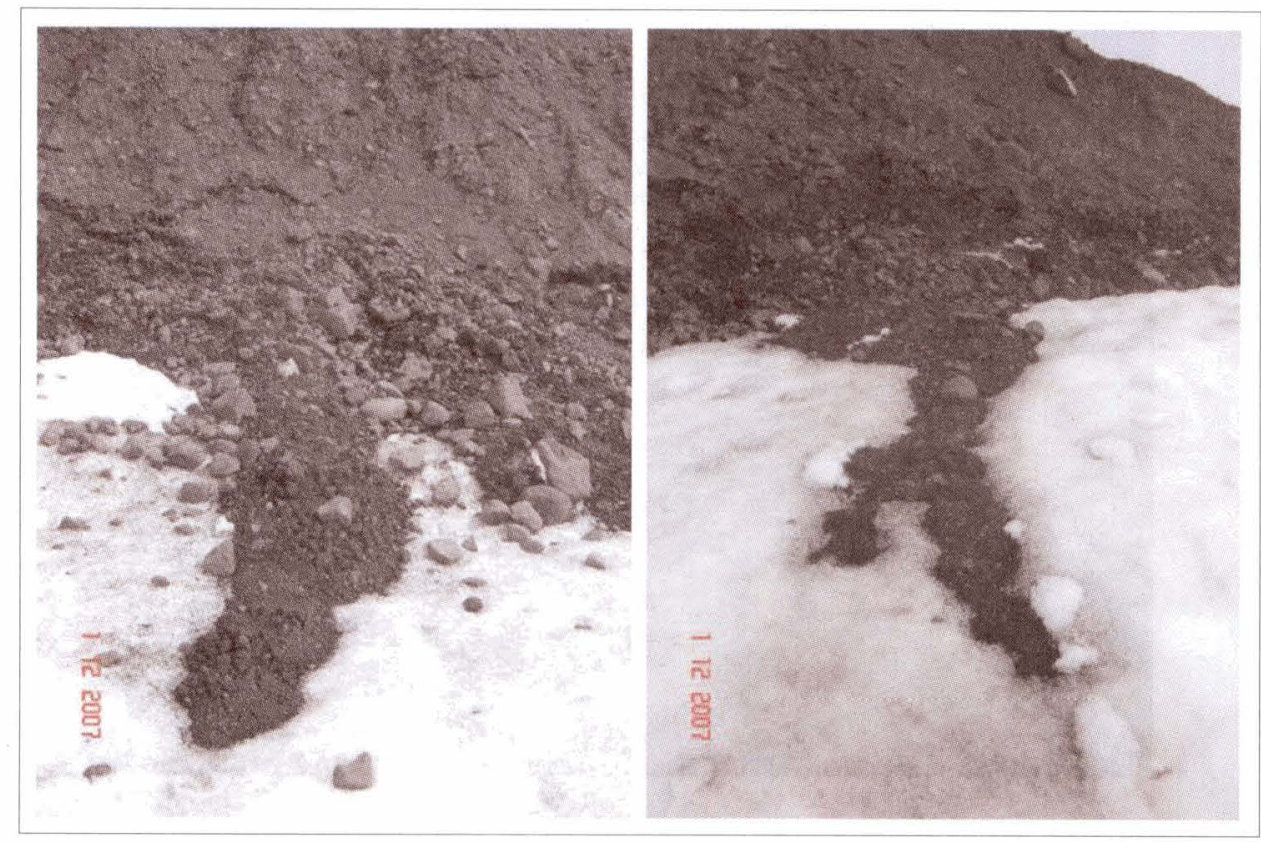

Fotos 10 y 11. Pequeños flujos detríticos en morrena lateral del Glaciar Krak (sector de Warkocs en Fig. 1) ante la ocurrencia de precipitaciones líquidas de algunas horas.

A ello se suma el retroceso de los frentes y aparecimiento de cuerpos lagunares entre el frente de hielo y los cordones morrénicos frontales, los cuales por acción del oleaje marino se encuentran rebajados o disectados, con lo que se han creado ambientes de lagunas salobres. En el caso del Glaciar Wanda, el cual antiguamente alcanzaba la costa de la Ensenada Martel (tidewater glacier) (Rosa et al., 2008a), el retroceso de su frente ha dejado desprovista de hielo una leve depresión post morrénica frontal cuyo ancho oscila entre 50 y $100 \mathrm{~m}$, la cual se encuentra ocupada por aguas de fusión del glaciar sumadas a aguas marinas que penetran por sobre el escaso y discontinuo relieve del depósito morrénico frontal debido a los efectos erosivos del oleaje y al ingreso de aguas costeras en condiciones de pleamar tanto como producto de tormentas y 'marejadas. (Foto 12)

Las dimensiones de estas lagunas permiten señalar que los frentes de estos glaciares han retrocedido por pérdida de masa desde varias decenas de metros hasta varias cen- tenas, como en el caso del Glaciar Ecology (Foto 13). Según Birkenmajer (2002), este glaciar ha estado retrocediendo durante los últimos 45 años. Los estudios de Vieira et al. (2005) indican que el retroceso del frente de este glaciar desde los años '70 al 2007 alcanza cerca de $400 \mathrm{~m}$.

\section{El apoyo sedimentológico}

Corroborando lo anterior, como parte de esta investigación, los estudios sedimentológicos de las muestras de los materiales dejados a la vista por el retroceso del Glaciar Wanda (ver Rosa et al., 2006; 2008a) revelan un abundante contenido de sedimentos finos y un cierto grado de redondeamiento, lo que indica que el régimen termal basal del glaciar es de carácter húmedo o politermal. Esta determinación se basa en el hecho que la ocurrencia de circulación basal de aguas de fusión y de lluvia provenientes de la superficie del glaciar produce desgaste de las aristas de los fragmentos durante el transporte subglacial, hecho que es característico en los detritos de las muestras analizadas. 


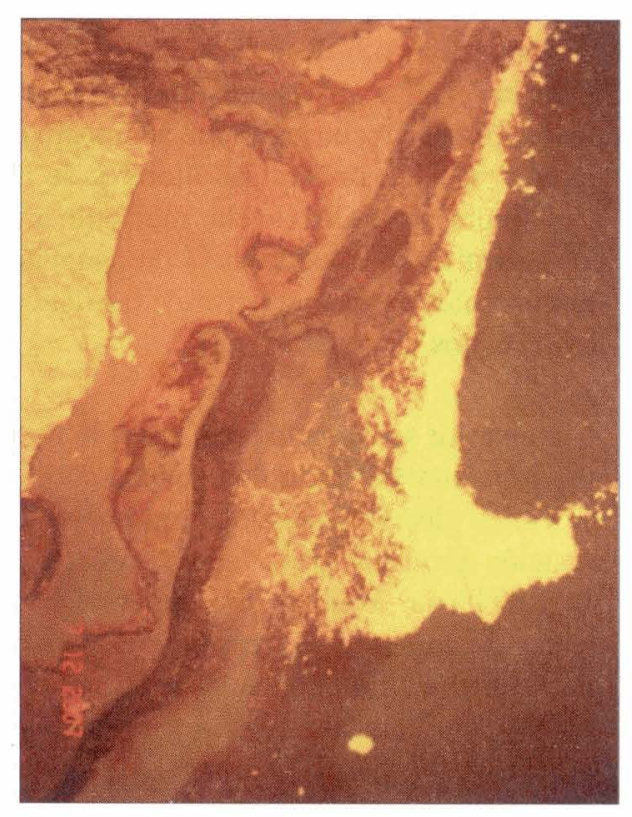

Foto 12. Sistema lagunar, con cordones morrénicos y playa de bloques cubierta parcialmente de nieve vieja y growlers, entre el frente del Glaciar Wanda y el mar. Se aprecian fondos lagunares secos detrás del cordón morrénico que indican una laguna mucho mayor antes del la ruptura de este.

(Sub-escena de una foto aérea de fines del verano polar 2006).

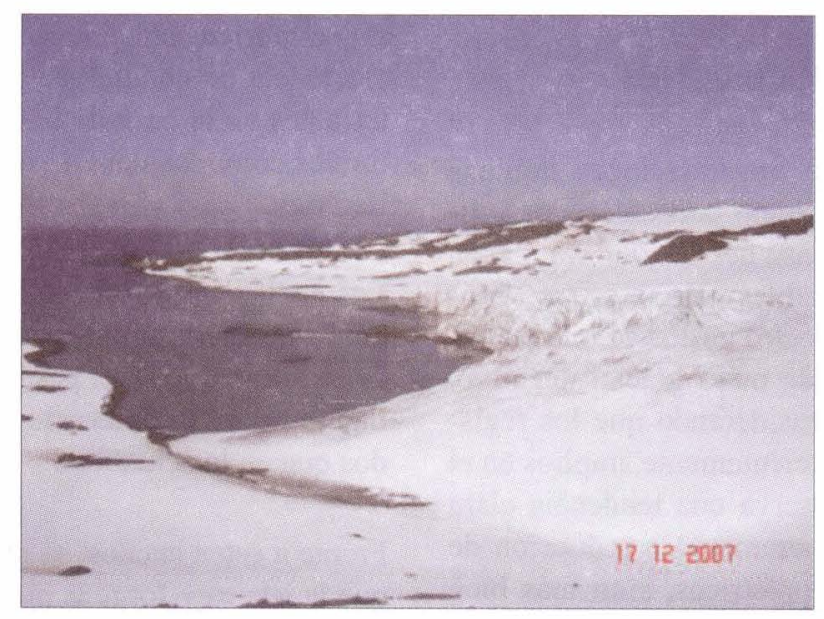

Foto 13. Frente del Glaciar Ecology y laguna de fusión separada del mar por un cordón morrénico desgastado y parcialmente segmentado por la acción del oleaje.

\section{El volcanismo como posible factor de fusión}

Debe tenerse presente que, según Hervé ét al. (2000), la evolución geológica de la Península Antártica durante el Meso-Cenozoico puede considerarse representativa de un arco plutónico-volcánico. Complementariamente, se reconocen manifestaciones tectónicas asociadas a la expansión que experimenta el Mar de Bransfield, las que entrañan fenómenos tanto geodinámicos como volcánicos (Berrocoso et al., 2008).

Teniendo en cuenta estos antecedentes y la existencia actual de volcanismo secundario activo (actividad hidrotermal en Isla Decepción), y las manifestaciones eruptivas históricas que se han determinado en las inmediaciones de la isla Rey Jorge, como 
es el caso de la isla Penguin (Birkenmajer, 1981), cabe suponer que la primera también podría presentar actividad magmática en su base considerando que, según Hervé et al. (2000), existe un ambiente tectónicamente distensivo acompañado de magmatismo alcalino. Dado ello, es apropiado suponer a priori algún efecto térmico en el sustrato rocoso de la isla (calor latente) que pudiera estar incidiendo en el régimen termal basal de la calota y los glaciares efluentes existentes en ella. Este supuesto teórico requiere, evidentemente, de los estudios correspondientes.

Es válido recordar aquí lo señalado por King y Comiso (2003) y por Turner et al. (2005) respecto del hecho que las tendencias más claras al incremento de las temperaturas se registran fundamentalmente en la península antártica y sus archipiélagos adyacentes.

\section{CONCLUSIONES}

La ocurrencia de precipitaciones líquidas sobre cuerpos de hielo peri-antárticos y litorales, como el caso del Glaciar Wanda, en proporciones variables pero, a su vez, considerables, es un claro gestor de una condición de balance de masa glacial negativo. $\mathrm{Al}$ respecto, y considerando que los registros no son lo suficientemente amplios en el tiempo, no se observa una tendencia clara en términos de incremento o reducción de los montos pluviométricos, sino más bien una distribución aleatoria.

Sin embargo, si se valida el hecho que hace más 30 años atrás no se registraba precipitación líquida en el área, es posible sostener un avance estacional significativo de la isoterma de $0^{\circ} \mathrm{C}$ hacia el sur durante las últimas décadas. Dicha aseveración se ve corroborada por las tendencias térmicas documentadas, las que vienen a explicar la desintegración de las plataformas de hielo en la Península Antártica, y la aceleración del movimiento de los glaciares alimentadores de ellas.
Esta situación se ve reflejada, también, en el adelgazamiento de las lenguas de hielo como la del Glaciar Wanda, y en el retroceso de los frentes, lo que ha estado acompañado de la formación de lagunas litorales de represa morrénica. Otro hecho morfodinámico indicativo es la ocurrencia de pequeños movimientos en masa a partir de los flancos de depósitos morrénicos.

A lo anterior se suma la formación de lagunas estacionales dentro de pequeña cubetas en valles glaciales dejados como áreas de nivación producto de la evolución recesiva del Glaciar Wanda, y la generación de cursos de agua que escurren supra y yuxta glaciarmente cada vez que se registran precipitaciones líquidas.

Los análisis sedimentológicos vienen a apoyar estas determinaciones y la ocurrencia de flujos hídricos subglaciales al demostrar la existencia de condiciones húmedas o politermales en la base de lenguas glaciales litorales como las del Gl. Wanda y la del Gl. Ecology.

Los hallazgos de vegetación en diferente grado de fosilización en la Isla Rey Jorge demuestran que en el pasado se ha desarrollado más de un intervalo tanto o más cálidos que en la actualidad.

Frente a estos hechos, se puede argumentar que la isla Rey Jorge y, por lo tanto, toda la parte norte de la Península Antártica y sus archipiélagos aledaños, han pasado por más de una oscilación climática en la que se han sucedido estadios a lo menos templados y lluviosos como parte de los períodos interglaciales, a tal punto benignos que han permitido el desarrollo de vegetación herbácea y leñosa. A este respecto, es posible argüir que la Península Antártica se podría encontrar en camino a una nueva era templada como las indicadas por las evidencias.

Finalmente, considerando los registros térmicos y lo señalado por investigaciones 
previas respecto a las tendencias climáticas en la península antártica, se plantea la interrogante del posible rol de emisiones de calor latente en la base de los glaciares, hecho asociado a cámaras magmáticas activas dados los antecedentes sobre manifestaciones volcánicas y magmatismo existentes, teoría que si bien tiene argumentos, carece de estudios específicos en este sentido.

\section{BIBLIOGRAFÍA}

ANTONIOLI, F. \& SILENZI, S. 2007. Variazioni relative del livello del mare e vulnerabilità delle pianure costiere italiane. Quaderni della Societa Geologica Italiana 2(2007): 2-29.

BERROCOSO, M.; ARIAS, M.R.; SERRANO, A.; FERNÁNDEZ-ROS, A.; DE GIL, A.; TORRECILLAS, C.; GARCÍA, J. A. 2008. Geodetic and thermometric monitoring of the volcanic activity at Deception Island (Antartica): VOLDETEC Project (2005-2008). En: IV Simposio Latinoamericano sobre Investigaciones Antárticas, Valparaíso, Chile. 382-384.

BIRKENMAJER, K. 1981. Raise marine features and glacial history in the vicinity of H. Arctowsky Station, King George Island (South Shetland Islands, Antartica). Quat. Sci Rev., 29(2):109-117.

BIRKENMAJER, K. 2002. Retreat of Ecology Glacier, Admiralty Bay, King George Island (South Shetland Islands, West Antartica), 1956-2001. Bulletin of Polish Academy of Sciences: Earth Sciences, 50(1):5-19.

CARRASCO, J.F., M. GONZÁLEZ, 2007. Climatología de la Península Antártica y de la Base Eduardo Frei Montalva. Editado y Publicado por la Dirección Me'teorológica de Chile, 118 pp.

FERRON, F. A.; SIMOES, J. C.; AQUINO, F. E. 2001. Série temporal de temperatura atmosférica para a Ilha Rei George, Antártica. Revista do Departamento de Geografia. São Paulo: USP 14: 25-32.
HERVÉ, F.; PARADA, M.; STOCKERT, B. 2000. Evolución tectónica de la Península Antártica e Islas Shetland del Sur basada en la obtención e interpretación de edades de trazas de fisión en Apatita y Circón. En: Antártica, Ciencia y Juegos, DID, Univ. de Chile, págs. 4 y 5 .

KING, J.C., 1994. Recent climate variability in the vecinity of the Antarctic Peninsula. Int. Journal of Climatology, 14, 357-369.

KING, J.C., COMISO, J. C. (2003). The spatial coherence of interannual temperatura variations in the Antarctic Peninsula. Geophysical Research Letter, 30(2), 1040.

KING, J.C., HARANGOZO, S.A. (1998). Climate change in the western Antarctic Peninsula 1945-present: observations and possible causes. Annals of Glaciology, 27, 571-575.

LAMBECK K.; ANTONIOLI F.; PURCELL A.; SILENZI S. (2004). Sea level change along the Italian coast for the past 10.000 yrs. Quaternary Science Reviews, 23, 1567-1598.

MARSHAL, G.J.; V. LAGUN, T.A. Y LACHLAN-COPE, 2002: Change in Antarcitic Peninsula tropospheric temperatures from 1956-99; a synthesis of observations and reanalysis data. Internatilnal Journal of Climatology, 22, 291-310.

MERCER, J, 1978. West Antarctic Ice Sheet and CO2 greenhouse effect: a threat of disaster. Nature, 271, 321 .

MEIER, M. F.; A. POST. 1987. Fast tidewater glaciers, J. Geophys. Res., 92, 9051-9058,

MOON, T.; I. JOUGHIN. 2008. J Changes in ice front position on Greenland's outlet glaciers from 1992 to 2007 . J. Geophys. Res., 113.

MÖRNER, N-A.; 1980. The Fennoscandian uplift: Geological data and their geodynamical implication. En Mörner (ed.): Earth Rheology, Isostasy and Eustasy. John Wiley \& Sons. 
POWELL, R. D. 1991. Grounding-line systems as second-order controls on fluctuations of tidewater termini of temperate glaciers. J. B. Anderson, G. M. Ashley (eds) Glacial marine sedimentation: Paleoclimate significance; Special Paper 261: 74-94, Denver: Geological Society of America.

ROSA, K.; VIEIRA, R.; SIMÕES, J. 2006. Dinâmica glacial e características sedimentares resultantes na zona proglacial da Geleira Ecology - Baía do Almirantado, Ilha Rei George - Antártica. Revista Brasileira de Geomorfologia 7(2): 51-60.

ROSA, K.; VIEIRA, R.; SIMÕES, J.; FERRANDO, F. 2008A. Interpretación de la evolución del ambiente de deglaciación del Glaciar Wanda, Isla Rey George, Antártica. IV Simposio latinoamericano sobre investigaciones Antárticas y VII Reunión Chilena de investigación Antártica. Valparaíso, 3-5 de septiembre, 2008.

ROSA, K.; VIEIRA, R.; FERRANDO, F.; SIMÕES, J. 2008B. Erosional landforms and deposition processes product of the deglaciation evolution at Wanda Glacier, King George Island. Conference SCAR/IASC IPY. Polar Research - Arctic and Antarctic Perspectives in the International Polar Year. July 8 - 11, St. Petersburg, Russia. 2008.

SANTANA, E.M.; DUMONT, J.F.; AZHAR, H. 2008. Boulder pavement in Greenwich Island, Antartic Peninsula: possible testimony of climate variation during the Holocene. En: IV Simposio Latinoamericano sobre Investigaciones Antárticas, Valparaíso, Chile. 442-446.

SIMOES, J. C.; BREMER, U. F.; AQUINO, F. E.; FERRON, F. A. 1999. Morphology and variations of glacial drainage basins in King George Island Icefield, Antartica. Annals of Glaciology, 29:220-224.
TORRES, T.; GALLEGUILLOS, H.; BARALE, G.; MEÓN, H.; PHILIPPE, M.; THEVENARD, F.; PRIEUR, A. 2000. Paleofloras del Meso-Cenozoico de las islas Shetland del Sur y Península Antártica. En: Antártica, Ciencia y Juegos, DID, Univ. de Chile, págs. 3 y 4.

TURNER, J.; S.R. COLWELL, G.J. MARSHALL, T.A. LACHLAN-COPE, A.M. CARLETON, P.D. JONES, V. LAGUN, P.A. REID S. IAGOVKINA, 2005. Antarctic climate change during the last 50 years. International Journal of Climatology, 25, 279-294.

VAUGHAN, D. G. 1993. Implications of the break-up of Wordie Ice Shelf, Antarctica for sea level. Antarctic Science, 5, 403408 .

VAUGHAN, D.G.; G.J. MARSHALL, W.M. CONNOLLEY, J.C. KING \& R. MULVANEY. 2001. Climate change: The devils in the details. Science, 293(5536): 1777-1779.

VAUGHAN, D.G.; G. MARSHALL, W. CONNOLLEY, C. PARKINSON, R. MULVANEY, D. HODGSON, J. KING, C. PUDSEY \& J. TURNER. 2003. Recent rapid regional climate warming on the Antarctic Peninsula. Climatic Change, 60(3): 243-274.

VIEIRA, R.; SUERTEGARAY, M.; AQUINO, F.; SIMOES, J. 2005. Feicoes morfologicas asociadas ao ambiente de deglaciacao da Geleira Ecology, Ilha Rey George, Antártica. Revista Brasileira de Geomorfología 6(2): 51-64.

YOON, H.H.; B. K. PARK, Y. KIM, D. KIM. 2000. Glaciomarine sedimentation and its paleo-oceanographic implications along the fjord margins in the South Shetland Islands, Antarctic, during the last 6000 years. Palaeog. Palaeocl. Palaeoec. Rev. 157: 189-211. 